Vol 6 No 1 February 2021

REVIEW ARTICLE

\title{
Liver Decellularization as Liver Organoid Reconstruction Scaffold
}

\author{
Christine Verawaty Sibuea \\ Faculty of Medicine, University of HKBP Nommensen \\ Correspondence email : christine.sibuea@yahoo.com
}

\begin{abstract}
The latest approach in tissue engineering techniques is the use of deselularized native organ as scaffolds for organ reconstruction. Scaffolds made from natural or synthetic matrix have many shortcomings in providing ideal extracellular matrix microstructure for cell proliferation and differentiation. Deselularized native scaffold can maintain the extracellular matrix of native organs, as an ideal microstructure scaffold for cell proliferation and differentiation. This technique answers the challenge of high need for liver transplants due to the limitations of liver donors and the need as liver models for drug testing.for drug testing. Liver is a complex organ, so ideal microstructure is needed in liver organoid reconstruction techniques to produce good synthetic and metabolic functions of the liver.
\end{abstract}

Keywords : Decellularization, scaffold, extracellular matrix, liver organoid

\section{INTRODUCTION}

The liver is a vital organ that has important functions in metabolism, protein synthesis and detoxification. The liver has a very good tissue regeneration ability so that damage to liver tissue can be repaired, but this does not occur when liver damage exceeds $90 \% .^{(1,2)}$ Continuous liver damage will result in cirrhosis and liver failure (liver decompensation). Cirrhosis is one of the top 10 causes of death in the world based on 2015 WHO data, which amounted to $20.3 \%$. According to the 2012 WHO data, the cirrhosis mortality rate in Indonesia is 52.7 (men) and 16.6 (women) per 100,000 deaths. ${ }^{(3)}$ The main therapy used in cirrhosis and terminal liver failure is liver transplantation. Cirrhosis, liver cancer, cholestatic disease, acute liver failure, and metabolic liver disease are diseases that will ultimately require a liver transplant. ${ }^{(4)}$ The increasing number of sufferers of the disease has resulted in an increased need for liver transplants. In 2016, the need for liver transplants in the US increased by 11,340 for adults and 723 for children. This high need for liver transplants is limited by the limited availability of donors, limited transplant expertise, and long-term use of immunosuppression. ${ }^{(3)}$

Various attempts were made to overcome the shortage of liver transplants. Alternative therapy with hepatocyte cell transplantation also has limitations due to the low engraftment of hepatocyte cells, which requires a very large number of 
hepatocyte cells, and the engrafted cells also do not develop and will still require a liver transplant. ${ }^{(5)}$ Another alternative therapy with a Liver-assist device (LAD) is only able to alleviate the sufferer's disease and stabilize the patient for one or two weeks until the organ is available for liver transplantation, but is unable to perform the detoxification and synthetic function of the liver permanently. ${ }^{(6)}$ The development of cell-based LAD also cannot be used because of the rapid decrease in hepatocyte cell viability, so that alternative therapies cannot be used for a long and permanent time. ${ }^{(6,7)}$

Tissue engineering techniques are still being developed to answer the high need for liver transplantation, namely liver regeneration using cell-based liver organoids. These liver organoids can also be used as a model of the liver for drug testing or to understand the mechanisms of liver disease. The need for liver organoids that resemble liver in vivo in microstructure and function poses challenges in the development of tissue engineering and refinement of methods. Liver organoids that use scaffold from natural or synthetic matrices cannot provide an ideal extracellular matrix microstructure for cell proliferation and differentiation. The desellularized liver scaffold maintains the extracellular matrix of the original organ so that it is a scaffold with an ideal microstructure for the proliferation and differentiation of liver cells. This article discusses liver deselularization, materials and methods of decellularization that are often used in tissue engineering for the reconstruction of liver organoids with the ideal microstructure with the liver in vivo.

\section{LIVER DECELLULARIZATION}

The purpose of the decellularization process is to eliminate cells and minimize damage to the extracellular matrix. The liver decellularization process removes hepatocytes and non-parenchymal cells, and maintains the extracellular matrix which will be used as a scaffold. The extracellular matrix on this scaffold has an ideal microstructure for the interaction between cells and the extracellular matrix, proliferation, differentiation and cell function. The original blood vessels can also be revascularized for oxygen and nutrient transport. ${ }^{(8)}$

The advantage of using scaffold from hepatic desellularization is the composition of the extracellular matrix and the structure of the original blood vessels, allowing for efficient recellularization. The cell mass can be adequately distributed throughout the organs and the cell has the right 3D microstructure attachment sites to develop into tissue. The scaffold of liver desellularization also has an extracellular matrix such as collagen types I and II, fibrin, fibtonectin, laminin, elastin-like polypetides, and polysaccharides such as hyaluronic acid, chitosan, and glycosamonoglycans (GSGs). Therefore the extracellular matrix of the desellularized scaffold is the most perfect material for cell adhesion, differentiation and proliferation. ${ }^{(9)}$

\section{MATERIAL AND METHODS LIVER DECELLULARIZATION}

There are several methods that are often used in liver deselularization, namely physical, enzymatic, and chemical methods. The choice of the desellularization method greatly affects the conservation of the extracellular matrix required for the reconstruction of liver organoids. Proper

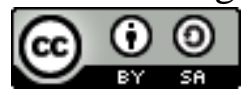


deselularization method will produce scaffold.

Chemical methods include using hypertonic sodium chloride, sodium hydroxide, sodium dodecyl sulfate (SDS), and Triton X-100. Recent decellularization studies often use SDS and Triton-X. SDS is a strong ionic solution and amphiphatic detergent, which can damage cell membranes and denature proteins. Triton X100 is a non-ionic detergent and can damage the interaction between lipids and lipids with proteins but does not damage interactions between proteins. ${ }^{(9)}$

Ren et al performed liver desellularization by portal perfusion using SDS and Triton X-100 solutions to dissolve cell membrane lipids. The liver was perfused with $1 \%$ SDS or $1 \%$ Triton $\mathrm{X}-100$ and $0.05 \%$ sodium hydroxide for 2 hours at a perfusion rate of $5 \mathrm{ml} / \mathrm{min}$. The liver is resellularized with $1.0 \quad-2.0 \quad$ x $10^{8}$ hepatocytes. The SEM examination showed Glissons capsule covering the portal vein and large vascular in the intact liver, and there was no significant difference between liver desellularization and $1 \%$ SDS or $1 \%$ Triton X-100. Immunohistochemical examination showed that type I collagen was seen between the lobules of the hepatocytes, type IV collagen was seen in the extracellular matrix surrounding the blood vessels, type $\mathrm{V}$ collagen was seen in the matrix near the vessels, and type VI collagen was seen in the hepatocyte area surrounding the blood vessels and there was no significant difference between desellularization of the liver with $1 \%$ SDS or $1 \%$ Triton X-100. Elastin in 1\% Triton X100 is more than $1 \%$ SDS, namely $20 \%$ and $60 \%$. GAGs and HGF were less at $1 \%$ SDS compared to using $1 \%$ Triton X-100. Albumin and urea production was greater at

Buletin Farmatera

Fakultas Kedokteran (FK)

Universitas Muhammadiyah Sumatera Utara (UMSU)

http://jurnal.umsu.ac.id/index.php/buletin_farmatera
$1 \%$ Triton X-100 compared to $1 \%$ SDS. Through this study, it can be concluded that Triton X-100 is more effective for liver desellularization. ${ }^{(10)}$

Cheng et al performed deselularization with Triton X-100 infused via a portal vein, and resellularization with Uygun's step-bystep perfusion culture of $100 \times 10^{6}$ HepG2 or C3A. SEM examination showed no residual cells and intact collagen. Western blot analysis showed that there was no difference between type I collagen, type IV collagen, fibronectin, and laminin between desellularized liver and fresh liver tissue. Cells grow well around the intrahepatic vascular and are distributed to the scaffold. Albumin production and urea synthesis showed a significant difference between perfusion culture and $2 \mathrm{D}$ culture in $\mathrm{C} 3 \mathrm{~A}$, but not in Hep G2. Liver function in desellularized liver perfusion culture is better than $2 \mathrm{D}$ culture. ${ }^{(11)}$

\section{LIVER DECELLULARIZATION IMPLEMENTATION}

The scaffold of hepatic decellularization can facilitate stem cell differentiation, and cell arrangement (spatial distribution). Hepatocytes are polarized cells, and this polarity is needed to maintain optimal cell conditions. In vivo hepatic extracellular matrix plays a role in maintaining cell function and cell polarity by maintaining a factor gradient so that hepatocyte phenotype can be maintained. Several researchers from various disciplines are collaborating to reconstruct liver organoids in vitro from complex cell components and extracellular matrix from liver desellularization. This liver organoid can be used as a Bioartificial Liver, thereby extending the waiting time for liver transplant patients; can be a measuring tool 
for drug toxicity studies; and increase understanding of the physiology of normal and damaged liver. ${ }^{(9)}$

The synthesis and metabolic functions of the liver can only be optimized by the growth of cells in a supportive matrix. This can be obtained using a scaffold of liver desellularization. Uygun et al performed liver deselularization and cultured hepatocytes in it. Hepatocytes cultured in the liver desellularize capable of performing specific liver functions such as albumin secretion, urea synthesis, and the expression of the metabolic enzymes of the drug. ${ }^{(2)}$

Mazza et al. Used intact human liver desellularization as an extracellular matrix scaffold with an intact architecture. Human liver desellularized scaffold was repopupopated for 21 days using human hepatic stellate cell line (LX2), hepatocellular carcinoma (Sk-Hep-1) and hepatoblastoma (HepG2). Cell viability and proliferation are excellent and extracellular matrix remodeling occurs. HE staining showed progressive proliferation and spread of LX2 into the scaffold within 21 days. Examination using SEM showed that LX2 migrates along the sinusoid, with the morphology of the fibroblasts being flat to spherical. This indicates mitosis. The extracellular matrix of the liver was also successfully repopulated with HepG2 that filled and migrated through the scaffold. ${ }^{(9)}$

Local environmental factors influence the homing, differentiation, and proliferation processes of hepatocytes. The interactions between cells and the surrounding extracellular matrix affect specific gene expression, morphogenesis, and cell migration. Artificial liver microstructure using tissue engineering and microfabrication techniques is one of the

Buletin Farmatera

Fakultas Kedokteran (FK)

Universitas Muhammadiyah Sumatera Utara (UMSU)

http://jurnal.umsu.ac.id/index.php/buletin_farmatera methods used to obtain these environmental factors, but this has not been successful so far, although many techniques and methods have been used. Synthetic biomaterials that are used as scaffold are limited in tissue regeneration and cell differentiation. The desellularized liver is a 3D scaffold with the right environment for multicellular in vitro growth. The extracellular matrix on the scaffold of liver decellularization will support viability, phenotype, function and hepatocyte tissue formation. ${ }^{(7)}$ Liver desellularization that was repopulated with LX2, HepG2 and Sk-Hep-1ber engraftment and migrated on the scaffold rapidly, with good viability and proliferation, motility and the occurrence of extracellular matrix remodeling. Repopulation of the scaffold with different liver cell types on the 3D scaffold structure allows the cells to homing and heading to the right location. This causes the liver desellularization scaffold to be used in drug testing and models to determine the mechanism of liver disease. ${ }^{(12)}$

\section{CONCLUSION}

Liver desellularization scaffold maintains the extracellular matrix of the original organ so that it is a scaffold with an ideal microstructure for cell proliferation and differentiation. This technique addresses the challenge of meeting the high need for liver transplantation given the limitations of the donor liver and the need for a liver model for drug testing.

\section{REFERENCES}

1. Li Y, Wu Q, Wang Y, Li L, Chen F, Shi Y, et al. Construction of bioengineered hepatic tissue derived from human umbilical cord mesenchymal stem cells via 
aggregation culture in porcine decellularized liver scaffolds. Xenotransplantation. 2017;24(1).

2. Uygun BE, Izamis ML, Jaramillo M, Chen Y, Price G, Ozer S, et al. Discarded Livers Find a New Life: Engineered Liver Grafts Using Hepatocytes Recovered From Marginal Livers. Artif Organs. 2017;41(6):579-85.

3. Kim WR, Lake JR, Smith JM, Schladt DP, Skeans MA, Harper AM, et al. OPTN/SRTS 2016 Annual Data Report : Liver. Scientific Registry of Transplant Recipients. 2016.

4. Blachier M, Leleu H, PeckRadosavljevic M, Valla DC, RoudotThoraval F. The burden of liver disease in Europe: a review of available epidemiological data. J Hepatol. 2013;58(3):593-608.

5. Hindley CJ, Cordero-Espinoza L, Huch M. Organoids from adult liver and pancreas: Stem cell biology and biomedical utility. Dev Biol. 2016;420(2):251-61.

6. Yagi H, Fukumitsu K, Fukuda K, Kitago M, Shinoda M, Obara H, et al. Human-scale whole-organ bioengineering for liver transplantation: a regenerative medicine approach. Cell Transplant. 2013;22(2):231-42.

7. Tamai M, Adachi E, Tagawa Y. Characterization of a liver organoid tissue composed of hepatocytes and fibroblasts in dense collagen fibrils. Tissue Eng Part A. 2013;19(2122):2527-35.

8. Baptista PM, Siddiqui MM, Lozier G, Rodriguez SR, Atala A, Soker S. The use of whole organ decellularization for the generation of a vascularized liver organoid. Hepatology. 2011;53(2):604-17.

9. Caralt M, Velasco E, Lanas A, Baptista PM. Liver bioengineering: from the stage of liver decellularized matrix to the multiple cellular actors and bioreactor special effects. Organogenesis. 2014;10(2):250-9.

10. Ren H, Shi X, Tao L, Xiao J, Han B, Zhang Y, et al. Evaluation of two decellularization methods in the development of a whole-organ decellularized rat liver scaffold. Liver Int. 2013;33(3):448-58.

11. Cheng Y, Wang Y, Kang YZ, Hu PY, Gao Y, Pan MX. In vitro culture of tumour-derived hepatocytes in decellularised whole-liver biological scaffolds. Digestion. 2013;87(3):18995.

12. Mazza G, Rombouts K, Rennie Hall A, Urbani L, Vinh Luong T, Al-Akkad $\mathrm{W}$, et al. Decellularized human liver as a natural 3D-scaffold for liver bioengineering and transplantation. Sci Rep. 2015;5:13079. 